\title{
Development of Enhanced Recovery Pathway for Patients Undergoing Lumbar Spine Surgery
}

\author{
Fassil B Mesfin ${ }^{1 *}$, Kris Coats $^{1}$ and Raul A Castillo ${ }^{2}$ \\ ${ }^{1}$ Division of Neurological Surgery, USA \\ ${ }^{2}$ Department of Neurosurgery, USA
}

*Corresponding author: Fassil B Mesfin, Associate Professor and Director of Spine Divsion of Neurological Surgery, MU School of

Medicine, Columbia, MO, USA

\section{ARTICLE INFO}

Received: 幽 July 15, 2019

Published: 㠈 July 23, 2019

Citation: Fassil B Mesfin, Kris Coats, Raul A Castillo. Development of Enhanced Recovery Pathway for Patients Undergoing Lumbar Spine Surgery. Biomed J Sci \& Tech Res 20(1)-2019. BJSTR. MS.ID.003381.

Keywords: Lumbar; Fusion; Surgery; Recovery

\begin{abstract}
Background: Enhanced recover after surgery (ERAS) pathways have been utilized to optimize patient in different surgeries. However, its implementation in lumbar spine surgery has been limited. ERAS pathways have been associated with beneficial patient outcomes, shorter hospital stays, and quicker recovery periods. Overall, ERAS pathways should minimize complications, reduce the length of stay, and improve the outcomes for lumbar spine surgery patients.
\end{abstract}

Objective: To enhance success and minimize recovery time for patients undergoing lumbar spine surgery by implementing an ERAS pathway.

Methods: This research is a retrospective review of lumbar spine surgery patients performed by a single surgeon at the University of Missouri Hospital over five years. Outcome measurements and demographics were retrieved from the electronic health record of each patient which included single-level and multi-level procedures.

Results: There were 323 total patients that were included in this retrospective review. 217 of the patients received a lumbar fusion procedure, 211 had a single-level procedure and 112 had a multi-level procedure. The single-level procedure length of stay (LOS), drain placement, and eventful hospital course differed significantly $(p<0.05)$ from the multilevel counterparts. The mean LOS for single-level patients was 2.2 days compared to 3.2 days for the multi-level group ( $\mathrm{p}<0.0001)$. Of these, $50.2 \%$ of single-level patients had a drain placed compared to $78.6 \%$ for the multi-level group ( $p<0.0001$ ). Patients who had a drain placed had a mean LOS of $3.1 \pm 1.7$, compared to a mean LOS of $1.7 \pm 1.3$ for patients who did not have a drain placed $(\mathrm{p}<0.0001)$. Lumbar fusion patients showed significant differences in LOS $(p<0.001)$ and drain placement $(p=0.01)$ between single and multilevel groups.

Conclusion: The ERAS pathway would be applicable to future lumbar spine surgery patients due to its ability to identify factors that could lead to increased LOS, estimated blood loss, and eventful hospital courses. However, due to the complexities of lumbar spine surgery, there are some limitations to its application. Further research and patient outcome data will be needed to determine the ERAS pathways' efficacy.

\section{Introduction}

Enhanced recovery after surgery (ERAS) is a multimodal, evidence-based approach that aims to modulate the surgical stress response to accelerate post-operative recovery and reduce morbidity. Acute and chronic lower back pain from lumbar degenerative disk disease is one of the leading causes of disability, morbidity and socioeconomic burdens globally among people over age 40 [1]. However, there is limited research that pertains to the use of the ERAS pathway in lumbar surgical patients. Concerns have developed regarding higher complication rates associated with multi-level lumbar procedures and fusions. Due to the lack of data, a retrospective study was conducted to identify whether there are differences in complications between single-level and multi-level lumbar spine surgery (LSS) patients as well as for patients that had 
a drain placed versus those patients that had not. Reviewing our data and outcomes, while incorporating a comprehensive number of evidence-based clinical studies, we propose the implementation of an ERAS pathway for all levels of LSS.

\section{Materials and Methods}

\section{Retrospective Data Review}

Following institutional review board approval, a retrospective review of clinical data was performed on patients that had undergone LSS by a single attending physician between the years of 2013 to 2018. Exclusion criteria were those with indications resulting from trauma, infection, or neoplasm. Primary outcome measurements included length of stay, complications, and 30-day readmission rates. Statistical analysis was performed via Microsoft Excel 2010 using unpaired 2-tailed t-tests with unequal variances. A p-value of $<0.05$ was defined as statistically significant.

\section{ERAS Pathway Design}

A literature review of established ERAS pathways was conducted using PubMed and the Journal of Neurosurgery for articles published before January 2019. The initial focus was broad, and subsequently narrowed to lumbar spinal procedures and fusions. We took note of one article in the Journal of Neurosurgery that utilized an ERAS pathway for lumbar surgery patients. They specifically made their own Enhanced Recovery After Spinal Surgery (ERSS) protocol, comparing the outcomes of patients who utilized the ERSS pathway and outcomes of patients who were preERSS. Based on the available evidence, recommendations were compiled and together with our outcome data, an ERAS protocol for our lumbar spine surgeries was proposed.

\section{Results}

\section{All Lumbar Spine Surgery Patients}

Single-level Procedure: For patients who underwent singlelevel LSS, there were 211 patients, $54 \%$ were males, with a mean age of 55.1 and a mean BMI of 31.2 (Table 1). On average, surgical time was 114.6 minutes, with an EBL of $83.9 \mathrm{~mL}$ and LOS of 2.2 days. 106 patients $(50.2 \%)$ had a surgical drain placed and 106 patients $(50.2 \%)$ were confirmed to have a history of smoking. Most patients had an unremarkable post-operative course requiring 1-3 days of admission but 20 patients (9.5\%) had eventful hospital stays, 7 of which were directly related to the procedure. One patient had a malpositioned screw that was causing severe discomfort, so the patient returned to the operating room after 3 days to have the screw properly positioned. Three patients had significant wound drainage that required the incision to be resewn or a Hemovac drain to be placed intraoperatively. Also, three patients had cerebral spinal fluid (CSF) leaks that were appropriately repaired intraoperatively. The remaining patients that had eventful hospital courses developed complications including: hypotension, hematuria, hyperglycemia, an ileus, and difficulty voiding. Only one patient (0.47\%) developed a wound infection that was successfully treated with a long-term course of antibiotics and hospital admission. Six patients (2.8\%) were readmitted within 30-days following discharge. One patient was admitted for a wound infection that was treated with a course of appropriate antibiotics. One patient was admitted for suspicion of a possible CSF leak, but was discharged the next day. Other patients were admitted for superficial cellulitis, extra removal of disk fragments, intestinal obstruction, and cholecystitis.

Table 1: All Lumbar Procedures.

\begin{tabular}{|c|c|c|c|}
\hline Parameter & Single level & Multi-level & p-value \\
\hline No. of patients & 211 & 112 & $<0.0001^{*}$ \\
\hline Mean age (years) & $55.1 \pm 13.1$ & $62.1 \pm 12.9$ & 0.22 \\
\hline Male sex (\%) & $113(53.6 \%)$ & $68(60.7 \%)$ & 0.19 \\
\hline BMI & $31.2 \pm 7.3$ & $32.3 \pm 7.1$ & 0.21 \\
\hline Smoking Hx (confirmed) & $106(50.2 \%)$ & $48(42.9 \%)$ & $<0.0001^{*}$ \\
\hline Drain & $106(50.2 \%)$ & $88(78.6 \%)$ & $<0.0001^{*}$ \\
\hline LOS (days) & $2.2 \pm 1.6$ & $3.2 \pm 1.7$ & $<0.0001^{*}$ \\
\hline EBL (mL) & $83.9 \pm 78.1$ & $162.8 \pm 117.4$ & $<0.0001^{*}$ \\
\hline Surgery Time (min) & $114.6 \pm 56.1$ & $161.2 \pm 55.6$ & $0.045^{*}$ \\
\hline Eventful hospital course & $20(9.5 \%)$ & $20(17.9 \%)$ & 0.68 \\
\hline Wound infection & $1(0.47 \%)$ & $1(0.89 \%)$ & 0.11 \\
\hline 30-day re-admission & $6(2.8 \%)$ & $8(7.1 \%)$ & \\
\hline
\end{tabular}

Note: Patient demographics and outcomes for single-level and multi-level lumbar spine procedures. Values are presented as the number of patients and percentages. This includes mean values \pm the standard deviation. $p-$ values are based off of a value of 0.05 for statistical significance. An “*” indicates a statistically significant value. BMI: body mass index. LOS: length of stay. EBL: estimated blood loss. 
Multi-Level Procedure: For patients who underwent multilevel LSS, there were 112 patients, $61 \%$ were males, with a mean age of 62 and a mean body mass index (BMI) of 32.3 (Table 1). On average, surgical time was 161 minutes, with an EBL of $162.8 \mathrm{~mL}$ and LOS of 3.2 days. 88 patients (78.6\%) had surgical drains placed and 48 patients (42.9\%) were confirmed to have a history of smoking. Most patients had an unremarkable post-operative course requiring 1-4 days of admission but 20 patients $(17.9 \%)$ had eventful hospital stays, 8 of which were directly related to the procedure. Five of these patients had significant wound drainage that required the incision to be resewn or a Hemovac drain to be placed intraoperatively. Also, three patients had a durotomy that was fixed intraoperatively. The other patients developed complications of urinary retention, urinary tract infections (UTIs), acute kidney injury, hyperglycemia, and an ileus. Only one patient $(0.89 \%)$ developed a wound infection that was properly treated with antibiotics. Eight patients (7.1\%) were readmitted within 30-days following discharge. One of the patients was admitted for a wound infection that was properly treated with a course of antibiotics. Two patients were also admitted and treated for adequate pain control. Other patients were admitted for severe constipation, altered mental status, hypotension, and diabetic ketoacidosis (DKA).

Patients with no Drain Placement: For patients who did not have a drain placed, there were 129 patients, 54\% were male, with a mean age of 54.7 and a mean BMI of 31.1 (Table 2). On average, surgical time was 94.8 minutes, with an EBL of $38.1 \mathrm{~mL}$ and LOS of 1.7 days. 57 patients $(44.2 \%)$ had a confirmed history of smoking. Most patients had an unremarkable post-operative course requiring 1-2 days of admission but 10 patients (7.8\%) had eventful hospital stays, 8 of which were directly related to the procedure. Four patients had significant wound leakage that was either resewn or extra dressing was applied. Another four patients had a durotomy or CSF leak that was repaired intraoperatively. The other two patients with eventful hospital stays were due to a mild rash and hyperglycemia. No patient developed a wound infection. However, three patients $(2.3 \%)$ were readmitted within 30-days following discharge. One of the patients was admitted for a suspected CSF leak, but was discharged the next day after appropriate testing was completed. Another patient needed extra disk fragments removed to alleviate severe discomfort and another patient was admitted to monitor superficial cellulitis.

Patients with Drain Placement: For patients who did have a drain placed, there were 194 patients, $57 \%$ were male, with a mean age of 59.4 and a mean BMI of 31.9 (Table 2). On average, surgical time was 154.7 minutes, with an EBL of $159.9 \mathrm{~mL}$ and LOS of 3.1 days. 97 patients (50\%) had a confirmed history of smoking. Most patients had an unremarkable post-operative course requiring 1-4 days of admission but 30 patients (15.5\%) had eventful hospital stays, five of which were directly related to the procedure. Three patients had significant wound leakage that was either resewn or monitored from the surgical drain. Another two patients had a durotomy that was repaired intraoperatively. Other complications included acute kidney injury, hematuria, ileus, constipation, urinary retention, UTI, increased need for oxygen, and hypotension. Two patients $(1.0 \%)$ developed wound infections and were treated with appropriate courses of antibiotics. Eleven patients $(5.7 \%)$ were readmitted within 30-days following discharge. Two of the readmissions were due to wound infections and were treated appropriately. Other reasons for readmission were pain control, hypotension, DKA, cholecystitis, constipation, and altered mental status.

Table 2: Drain Placed vs No Drain.

\begin{tabular}{|c|c|c|c|}
\hline Parameter & No Drain & Drain & p-value \\
\hline No. of patients & 129 & 194 & $0.004^{*}$ \\
\hline Mean age (years) & $54.7 \pm 16.0$ & $59.4 \pm 11.0$ & 0.6 \\
\hline Male sex (\%) & $70(54.3 \%)$ & $111(57.2 \%)$ & $<0.0001^{*}$ \\
\hline LOS (days) & $1.7 \pm 1.3$ & $3.1 \pm 1.7$ & 0.37 \\
\hline BMI & $31.1 \pm 7.3$ & $31.9 \pm 7.3$ & $<0.0001^{*}$ \\
\hline EBL (mL) & $38.1 \pm 43.8$ & $159.9 \pm 98.5$ & $<0.0001^{*}$ \\
\hline Surgery Time (min) & $94.8 \pm 63.7$ & $154.7 \pm 43.6$ & $<0.0001^{*}$ \\
\hline Number of levels & $1.3 \pm 0.7$ & $97(50.0 \%)$ & 0.31 \\
\hline Smoking Hx (confirmed) & $57(44.2 \%)$ & $30(15.5 \%)$ & $0.03^{*}$ \\
\hline Eventful hospital course & $10(7.8 \%)$ & $2(1.0 \%)$ & 0.16 \\
\hline Wound infection & $0(0.0 \%)$ & $11(5.7 \%)$ & 0.12 \\
\hline 30-day re-admission & $3(2.3 \%)$ & & \\
\hline
\end{tabular}

Note: Patient demographics and outcomes for patients with and without a surgical drain placed. Values are presented as the number of patients and percentages. This includes mean values \pm the standard deviation. $p$ - values are based off of a value of 0.05 for statistical significance. An “*” indicates a statistically significant value. LOS: length of stay. BMI: body mass index. EBL: estimated blood loss. 


\section{Lumbar Fusion Patients}

Single-Level Procedure: For patients who underwent singlelevel lumbar fusion surgery, there were 132 patients, 51\% were male, with a mean age of 57.5 and a mean BMI of 30.8 (Table 3). On average, surgical time was 145 minutes, with an EBL of 120.2mL and LOS of 2.6 days. 102 patients (77.3\%) had a surgical drain placed and 72 patients (54.5\%) had a confirmed history of smoking. Most patients had an unremarkable post-operative course requiring 1-3 days of admission but 14 patients (10.6\%) had eventful hospital stays, 4 of which were directly related to the procedure. One patient had a malpositioned screw which was surgically corrected. Two patients had incision drainage that was either resewn or maintained with a Hemovac drain. Another patient had a durotomy with CSF leak that was repaired intraoperatively. Other complications included hypotension, hematuria, acute kidney injury, ileus, urinary retention, and increased need for oxygen. One patient $(0.75 \%)$ developed a wound infection and was treated with appropriate antibiotics. Three patients $(2.3 \%)$ were readmitted within 30-days following discharge. One readmission was due to a wound infection, the other readmissions were due to intestinal obstruction and cholecystitis.

Table 3: Lumbar Fusion Procedures.

\begin{tabular}{|c|c|c|c|}
\hline Parameter & Single level & Multi-level & p-value \\
\hline No. of patients & 132 & 85 & \\
\hline Mean age (years) & $57.5 \pm 11.4$ & $61.4 \pm 12.0$ & $0.02^{*}$ \\
\hline Male sex (\%) & $67(50.8 \%)$ & $51(60.0 \%)$ & 0.18 \\
\hline BMI & $30.8 \pm 7.1$ & $32.4 \pm 7.1$ & 0.12 \\
\hline Smoking Hx (confirmed) & $72(54.5 \%)$ & $43(50.6 \%)$ & 0.57 \\
\hline Drain & $102(77.3 \%)$ & $76(89.4 \%)$ & $0.01^{*}$ \\
\hline LOS (days) & $2.6 \pm 1.5$ & $3.4 \pm 1.7$ & $<0.001^{*}$ \\
\hline $\mathrm{EBL}(\mathrm{mL})$ & $120.2 \pm 77.3$ & $188.4 \pm 118.7$ & $<0.0001^{*}$ \\
\hline Surgery Time (min) & $145.5 \pm 45.8$ & $177.0 \pm 49.8$ & $<0.0001^{*}$ \\
\hline Eventful hospital course & $14(10.6 \%)$ & $16(18.8 \%)$ & 0.11 \\
\hline Wound infection & $1(0.75 \%)$ & $1(1.1 \%)$ & 0.77 \\
\hline 30-day re-admission & $3(2.3 \%)$ & $7(8.2 \%)$ & 0.07 \\
\hline
\end{tabular}

Note: Patient demographics and outcomes for single-level and multi-level lumbar spine fusion procedures. Values are presented as the number of patients and percentages. This includes mean values \pm the standard deviation. $p-$ values are based off of a value of 0.05 for statistical significance. An “*” indicates a statistically significant value. BMI: body mass index. LOS: length of stay. EBL: estimated blood loss.

Multi-Level Procedure: For patients who underwent multilevel lumbar fusion surgery, there were 85 patients, $60 \%$ were male, with a mean age of 61.4 and a mean BMI of 32.4 (Table 3). On average, surgical time was 177 minutes, with an EBL of $188.4 \mathrm{~mL}$ and LOS of 3.4 days. 76 patients (89.4\%) had a surgical drain placed and 43 patients (50.6\%) had a confirmed history of smoking. Most patients had an unremarkable post-operative course requiring 1-4 days of admission but 16 patients (18.8\%) had eventful hospital stays, 5 of which were directly related to the procedure. Two patients had incision drainage that was either resewn or maintained with a Hemovac drain. The other three patients had a durotomy that was fixed intraoperatively. Other complications included hyperglycemia, increased need of oxygen, acute kidney injury, urinary retention, and UTI. One patient (1.1\%) developed a wound infection that was treated with appropriate antibiotics. Seven patients (8.2\%) were readmitted within 30-days following discharge. One of the readmissions was a patient with a wound infection that needed to be treated. Other complications that resulted in readmission included: pain control, DKA, an ileus, severe constipation, and hypotension.

\section{Discussion}

A retrospective review of our clinical data over the last 5 years demonstrates a pattern of practice that is amenable to implementation of an ERAS protocol that can reduce hospital length of stay from multiple overnight stays to a single overnight stay. For all levels of LSS, most of our patients are currently being kept overnight and some are kept for multiple nights. Nevertheless, we try to make minimal use of drains and keep the surgical time as short as possible. Our data (Table 2) shows that there is a significant difference between the LOS of patients that have drains placed compared to those who do not have drains placed $(\mathrm{p}<0.0001)$. This correlation is one of the reasons we believe that the proposed ERAS pathway can be applied to all levels of LSS while reducing the LOS and improving outcomes.

\section{Preoperative}

The preoperative component of the ERAS pathway aims to optimize the patient physical and functional status and to educate the patient about the surgical procedures and recovery [2]. 
Surgical Education: Patient surgical education should include pre-admission testing, medication use, peri-operative eating and drinking, postoperative expectations, instructions before surgery and postoperative follow-up procedures [2]. Surgical intervention is generally regarded as the last resort in alleviating patient discomfort and neurological symptomology after physical therapy and pain management attempts have failed. Many patients receive an epidural steroid injection (ESI) before undergoing LSS, but nearly one in six undergo surgery within a year following an ESI [3]. A retrospective study showed a significant increase in infection rates between patients who received an ESI within 3 months of lumbar surgery and patients those waited longer than 3 months before surgery [4]. This particular study only included patients that underwent a single-level lumbar procedure; however, the data suggests that surgery should be scheduled at least 3 months past the date of the ESI to minimize infection risk. In addition, patients should be provided with surgical site cleansing education. A chlorohexidine gluconate ( $\mathrm{CHG}$ ) solution should be provided for washing the incision site for three consecutive nights before surgery and the morning of [2]. The rate of compliance can be monitored by asking the patients about CHG use on the day of surgery.

Opioid use: There is particular concern of opioid use, its efficacy in pain management and prior or subsequent dependence for patients who undergo LSS $[5,6]$. It has been shown that opioid use is associated with less favorable outcomes following spinal surgery, such as increased LOS, lumbar fusion complications, pain management and higher cost [7]. This study concluded that patients who were opioid dependent had 2 times higher odds of a longer LOS, associated higher hospital cost and more likely to continue opioid use following discharge [7]. A different study focused on the length of time patients continue using opioids after spinal fusion surgery if they had used opioids beforehand; of those patients who received long-term opioids before lumbar surgery, $77.1 \%$ continued longterm postoperative use, $13.8 \%$ displayed episodic postoperative use and only $9.1 \%$ stopped or had short-term postoperative use [8]. In order to optimize patient outcomes, reduce cost and LOS, and avoid worsened postoperative pain, it is important to encourage patients to cease opioid use before lumbar surgery if possible, however, simple opioid reduction or cessation is unlikely without provision of viable alternatives to pain management, coping mechanisms and appropriate treatment programs [9].

Diabetes: In patients undergoing LSS having either insulindependent diabetes mellitus (IDDM) and non-insulin-dependent diabetes mellitus (NIDDM) are associated with increased risk of surgical site infections, wound dehiscence increased LOS and other complications [10]. Further, diabetes is a comorbidity that is known risk factor for reoperation, promotes spinal stenosis, increases disk degeneration and often results in poorer patient outcomes [11]. A 2014 retrospective study was conducted to determine what postoperative complications occurred after LSS in IDDM and NIDDM patients, this study found that IDDM patients showed a greater risk of death, septic shock, stroke, renal failure, greater LOS, pneumonia, readmittance and others, while NIDDM was associated with a risk of wound dehiscence and longer LOS [10]. It is important to monitor and educate patients on controlling their diabetes before undergoing LSS. Pre-operative Hemoglobin $\mathrm{A} 1 \mathrm{C}(\mathrm{HbA} 1 \mathrm{c})$ is a reliable measure of chronic diabetes control, so patients with $\mathrm{HbA} 1 \mathrm{c}>8 \%$ or a random serum glucose $>200 \mathrm{mg} /$ $\mathrm{dL}$ should have an endocrinology consult to achieve better glycemic control [2].

Nutrition: Nutritional optimization is an essential component of preoperative preparation. Beyond maintaining a healthy BMI, patients should be informed of the benefits of a balanced diet before undergoing LSS. A recent study found that malnutrition increases the LOS, infection rate, wound dehiscence and slows the healing process [12]. Many studies have been conducted relating BMI to the success rate of LSS and corresponding complications to patients with a high BMI. One study concluded that a greater surgical success rate was achieved when a BMI cut-off value of $\leq 29.1$ was applied as a predictive factor [13]. Another retrospective study showed that morbidly obese (BMI $\geq 35$ plus two major comorbid factors) or (BMI $\geq 40$ ) patients showed a significantly higher perioperative and postoperative complication rates [14]. The high rates of comorbidity and other health issues that are involved with obesity make it difficult to identify a pure correlation in LSS complications, and while no evidence exists suggesting that LSS is unsafe past a specific BMI, evidence does exist which suggests BMI is a factor in LSS success rates.

Smoking: Smoking cessation is highly advisable. Recent studies have found that smoking cessation improves outcomes regarding pain, progress after surgery, decreased infection risk and postoperative satisfaction [2]. Contradictory, a retrospective analysis show there seems to be no statistically significant difference between fusion rates of smokers and non-smokers and showed no association of poor clinical outcomes with smoking [15], while other reports significantly lower rates of successful fusion for smokers [16]. There is also research that recommends lumbar fusion surgery patients to remain non-smokers at least 4 weeks after surgery because an immediate return to smoking will inhibit vital angiogenesis, thus slowing fusion [17]. Currently, our standard of practice for active smokers is they must completely stop smoking 4-weeks prior to surgery with appropriate aids and counseling as part of the ERAS pathway.

Anxiety and Depression: Depression and anxiety is more common in-patient populations that are exposed to chronic pain [18]. Patients with a greater degree of depression have been shown to have worse postoperative outcomes than patients without depression [19]. Of those patients who have depression, there is a small decrease in depressive symptoms after LSS [19]. This helps support the idea that chronic pain can affect the severity of depressive symptoms, while depressive symptoms 
can worsen physical outcomes. Anxiety seems to show the same pattern as depression in patients who undergo LSS and can be a stronger factor in patients with preoperative fears. More research is necessary regarding LSS and psychological health, but patients with an established psychiatric diagnosis should be ensured treatment compliance prior to surgery to optimize recovery in this ERAS pathway.

\section{Intraoperative}

The intraoperative period begins at the start of surgery and ends with patient transfer to the Post-Anesthesia Care Unit (PACU). The focus is to optimize anesthetic effects and surgical procedures to ensure optimal convalescence.

Anesthesia: Oral pre-emptive analgesia to improve postoperative nausea should be provided in the preoperative holding area [9]. Treatment should include acetaminophen (1g), gabapentin (300mg), and scopolamine patch (1.5mg). Total intravenous anesthesia techniques will be used preferentially while minimizing intravenous opioids [20]. Antibiotic prophylaxis should also be administered at this time. To reduce recurrent laryngeal nerve palsy, the anesthesiologists, following placement of surgical retractors, should deflate the endotracheal cuff and then re-inflate until a seal is obtained and when possible, reduce the endotracheal cuff pressure to below $20 \mathrm{~mm} \mathrm{Hg} \mathrm{[21].}$

Steroid: Intravenous dexamethasone $(0.2 \mathrm{mg} / \mathrm{kg})$ will be given prior to surgical incision to reduce post-operative discomfort. A recent retrospective study shows that intravenous steroid application carries less of risk of post-operative infection than epidural steroid application, thus decreasing the risk of extended LOS and further complications, while both methods have been proven effective in reducing postoperative pain [22].

Foley Catheter Use: Postoperative urinary retention is highly variable among LSS patients [23]. Patients undergoing LSS are encouraged to void prior to anesthesia and the decision to place a Foley catheter is made on a case-by-case basis. A retrospective study found that the type of lumbar surgery, number of levels, surgical time and EBL were not associated with postoperative urinary retention [24]. If the decision is made to place a Foley catheter, it should be removed as soon as medically possible to decrease the chances of a UTI, decrease the LOS and lower the risk of other complications [23]. Avoiding the use of a Foley catheter is the preferred course of care as it encourages early mobilization and rehabilitation efforts.

Early Start Time: Ignoring out-patient procedures, many LSS patients stay in the hospital overnight. However, it is good clinical practice to schedule the surgery early in the day to optimize the chances of the patient being discharged sooner. Early start times will encourage shorter LOS and decrease hospital stay cost.

Neurological Monitoring: Intraoperative neurological monitoring will not be used on LSS patients. A recent retrospective study has shown that neurological monitoring during LSS may not decrease the chance of post-operative neurological complications significantly [25]. This same study stated that there is even evidence of false negatives in neurological monitoring, and this will not help surgeons decrease the chances of a neurological accident [25]. Another paper published in the Journal of Neurosurgery stated that there is no evidence that neurological monitoring can alter the outcomes of lumbar fusion patients [26].

Surgical Drain: It is the judgement of the surgeon to place a surgical drain, which will be made on a case-by-case basis. There is a lack of evidence that associates surgical drain usage with postoperative infections and complications. A systematic review of lumbar spine patients with closed suction drains concluded that surgeons should not regularly rely on drains until more conclusive evidence becomes available supporting the use [27]. Despite a lack of current evidence, a paper from 2012 did show that there appears to be no significant difference in the formation of a hematoma postoperatively between patients with a drain placed and those without [28]. The same study found no significant difference in the rates of postoperative infections or LOS [28]. This information is consistent with our data (Table 2) that there is no significant difference in wound infections between patients that had drains placed and those who did not ( $p=0.16)$. Ultimately, it is the surgeon's clinical judgement and ERAS understanding to determine if a drain is necessary for the patient.

\section{Postoperative}

The postoperative period includes care in the PACU and follow-up after discharge. Emphasis is placed on direct patient communication after leaving the hospital to ensure continuity of care and rehabilitation.

Pain Control: Postoperative pain control will employ preemptive and multimodal analgesia instead of patient-controlled analgesia and avoid the use of intravenous opioid administration. By inhibiting central autonomic hyperactivity with the use of nonsteroidal anti-inflammatory drugs, anti-convulsant, and acetaminophen, pre-emptive analgesia has shown improvement in immediate post-operative pain, anxiety and self-care [29]. Recommended practice is to use $600 \mathrm{mg}$ gabapentin immediately postoperatively and $975 \mathrm{mg}$ acetaminophen every 6-hours, unless a patient has known renal or hepatic dysfunction [2]. Oral narcotics should be used on a needed basis, while intravenous narcotics will be minimized. Patient controlled analgesia will not be used as multimodal analgesia has shown to control postoperative pain significantly better than intravenous narcotics [30].

Follow-up communication: After discharge from the hospital, a member of the surgical team should call the patient within 48-hours to discuss pain management, diet, mobility, wound care, and available triage resources for any concerns and follow-up instructions [2]. The patient will should up with both the primary care physician and surgeon within 2 weeks and again in 3 months. 
Clinical monitoring and surveys regarding outcomes will be administered during these visits.

\section{Conclusion}

The goal of the proposed ERAS pathway is to minimize patients LOS, decrease the risk of complications and encourage optimum patient outcomes. Besides being a patient-centered approach and modulating the stress of the surgical procedure, the ERAS pathway is also an iterative improvement process that allows for quick and efficient change to each step of the protocol based on outcome data [31]. The success of this protocol will depend on establishing a core team of nurses, surgeons, anesthesiologists and ancillary staff, as well as having a receptive institutional culture and the availability of resources. Importantly, prospective outcome measurements need to be adequately powered and reviewed on a regular basis to incorporate incremental protocol changes.

\section{Disclosure}

The authors report no conflict of interest concerning the materials or methods used in this study or the findings specified in this paper.

\section{References}

1. Olalekan AL, Alimi M, Gbadegesin SA, Muhammad O (2018) Treatment outcome of quality of life and clinical symptoms in patients with symptomatic lumbar degenerative disc disease: which treatment modality is superior? Int Orthop 1-7.

2. Ali ZS, Ma TS, Ozturk AK, Malhotra NR, Schuster JM, et al. (2018) Preoptimization of spinal surgery patients: Development of a neurosurgical enhanced recovery after surgery (ERAS) protocol. Clin Neurol Neurosurg 164: $142-153$.

3. Koltsov JCB, Smuck MW, Zagel A, Alamin TF (2018) Lumbar epidural steroid injections for herniation and stenosis: incidence and risk factors of subsequent surgery. Spine J 1-7.

4. Yang S, Werner BC, Cancienne JM, Hassanzadeh H (2015) Preoperative epideral injections are associated with increased risk of injection after single-level lumbar decompression. Spine J 16: 191-196.

5. Connolly III J, Javed Z, Raji M, Chan W (2017) Predictors of long-term opioid use following lumbar fusion surgery. Spine (Phila Pa 1976) 42: $1405-1411$.

6. Kim S, Ha K, Oh I (2015) Preemptive multimodal analgesia for postoperative pain management after lumbar fusion surgery: a randomized controlled trial. Eur Spine J 25: 1614-1619.

7. Tank A, Hobbs J, Ramos E, Rubin DS ( 2018) Opioid dependence and prolonged length of stay in lumbar fusion: A retrospective study utilizing the national inpatient sample 2003-2014. Spine 43: 1739-1745.

8. Deyo RA, Hallvik SE, Hildebran C, Marino M ( 2018) Use of prescription opioids before and after an operation for chronic pain (lumbar fusion surgery). Pain 159: 1147-1154.

9. Mc Anally H (2017) Rationale for and approach to preoperative opioid weaning: a preoperative optimization protocol. Perioper Med (Lond) 6 : 19.

10. Golinvaux NS, Varthi AG, Bohl DD, Basques BA (2014) Complication rates following elective lumbar fusion in patients with diabetes: Insulin dependence makes a difference. Spine 39: 1809-1816.

11. Kim CH, Chung CK, Shin S, Choi BR (2015) The relationship between diabetes and the reoperation rate after lumbar spinal surgery: A nationwide cohort study. Spine J 15: 866-874.
12. Puvanesarajah V, Jain A, Kebaish K, Shaffrey C (2017) Poor nutrition status and lumbar spine fusion surgery in the elderly: Readmissions, complications, and mortality. Spine 42: 979-983.

13. Azimi P, Yazdanian T, Shahzadi S, Benzel EC (2018) Cut-off value for body mass index in predicting surgical success in patients with lumbar spinal canal stenosis. Asian Spine J 12: 1085-1091.

14. Epstein NE (2017) More risks and complications for elective spine surgery in morbidly obese patients. Surg Neurol Int 8-66.

15. Echt M, Ramos RDG, Nakhla J, Gelfand Y (2018) The effect of cigarette smoking on wound complications after single-level posterolateral and interbody fusion for spondylolisthesis. World Neurosurg 116: 824-829.

16. Phan K, Fadhil M, Chang N, Giang G (2018) Effect of smoking status on successful arthrodesis, clinical outcome, and complications after anterior lumbar interbody fusion (ALIF). World Neurosurg 110: 9981003.

17. Berman D, Oren JH, Bendo J, Spivak J (2017) The effect of smoking on spinal fusion. Int J Spine Surg 11: 229-238.

18. Ghoneim MM, O’Hara MW (2016) Depression and postoperative complications: an overview. BMC Surg 16.

19. Merrill RK, Zebala LP, Peters C, Qureshi SA (2018) Impact of depression on patient-reported outcome measures after lumbar spine decompression. Spine (Phila Pa 1976) 15: 434-439.

20. Soffin EM, Vaishnav AS, Wetmore D, Barber L, Hill P, et al. (2018) Design and implementation of an enhanced recovery after surgery (ERAS) program for minimally invasive lumbar decompression spine surgery: Initial experience. Spine (Phila Pa 1976).

21. Jung A, Schramm J (2010) How to reduce recurrent laryngeal nerve palsy in anterior cervical spine surgery: a prospective observational study. Neurosurgery 67:10-15; discussion 15 .

22. Hu A, Gu X, Guan X, Fan G (2018) Epidural versus intravenous steroids application following percutaneous endoscopic lumbar discectomy. Medicine 97.

23. Golubovsky JL, Ilyas H, Chen J, Tanenbaum JE (2018) Risk factors and associated complications for postoperative urinary retention after lumbar surgery for lumbar spinal stenosis. Spine J 18: 1533-1539.

24. Gandhi SD, Patel SA, Maltenfort M, Anderson DG (2014) Patient and surgical factors associated with posteroperative urinary retention after lumbar spine surgery. Spine 39: 1905-1909.

25. Ajiboye RM, Zoller SD, D'Oro A, Burke ZD (2017) The utility of intraoperative neuromonitoring for lumbar pedicle screw placement is questionable: A review of 9957 cases. Spine (Phila Pa 1976) 42: 10061010.

26. Sharan A, Groff MW, Dailey AT, Ghogawala Z (2014) Guideline update for the performance of fusion procedures for degenerative disease of the lumbar spine. Part 15: Electrophysiological monitoring and lumbar fusion. Journal Neurosurg 21: 102-105.

27. Waly F, Alzahrani MM, Abduljabbar FH, Landry T (2015) The outcome of using closed suction wound drains in patients undergoing lumbar spine surgery: A systematic review. Global Spine J 5: 479-485.

28. Walid MS, Abbara M, Tolaymat A, Davis JR (2012) The role of drains in lumbar spine fusion. World Neurosurg 77: 564-568.

29. Lee BH, Park JO, Suk KS, Kim TH, Lee HM, et al. (2013) Pre-emptive and multi-modal perioperative pain management may improve quality of life in patients undergoing spinal surgery. Pain Physician 16: 217-226.

30. Pinar HU, Omer K, Karakoc F, Dogan R (2017) Effects of addition of preoperative intravenous ibuprofen to pregabalin on postoperative pain in posterior lumbar interbody fusion surgery. Pain Res Manag.

31. Wang MY, Chang PY, Grossman J (2017) Development of an Enhanced Recovery After Surgery (ERAS) approach for lumbar spinal fusion. J Neurosurg Spine 26: 411-418. 


\section{ISSN: 2574-1241}

DOI: 10.26717/BJSTR.2019.20.003381

Fassil B Mesfin. Biomed J Sci \& Tech Res

CC This work is licensed under Creative

Submission Link: https://biomedres.us/submit-manuscript.php

$\begin{array}{ll}\text { BIOMEDICAL } & \text { Assets of Publishing with us } \\ \text { RESEARCHES } & \text { - Global archiving of articles } \\ \text { - Immediate, unrestricted online access } & \text { - Rigorous Peer Review Process } \\ & \text { - Authors Retain Copyrights }\end{array}$

